\section{Breast lesions during pregnancy and lactation: a pictorial essay}

\author{
Seung Eun Lee', Young Kyung Bae² \\ Departments of ${ }^{1}$ Radiology and ${ }^{2}$ Pathology, Yeungnam University College of Medicine, \\ Daegu, Korea
}

A wide range of breast lesions can arise during pregnancy and lactation, from benign or inflammatory diseases to malignant tumors. Hormone-influenced physiological changes of the breast make the radiological evaluation of breast lesions challenging. Knowledge of the imaging features of normal physiological changes and common breast lesions during this period can help radiologists accurately diagnose and appropriately manage conditions. As such, this pictorial essay illustrates normal physiological changes related to pregnancy and lactation, as well as common benign and malignant breast lesions encountered during those periods.

Keywords: Breast diseases; Pregnancy; Lactation; Mammography; Ultrasound

\section{Introduction}

During pregnancy and lactation, the breast undergoes physiological changes under the influence of various hormones. These manifest as parenchymal tissue pattern alterations and increased blood flow [1]. Changes of this nature may make the radiological evaluation of breast lesions more difficult $[2,3]$. Most breast lesions detected during pregnancy and lactation are benign; however, the possibility of pregnancy-associated breast cancer (PABC) must be considered to avoid a delayed diagnosis [4]. The purpose of this article is to review the imaging findings associated with pregnancy- and lactationrelated physiological changes of the breast and to discuss the benign and malignant lesions common during those periods.

\section{Normal Physiological Changes of the Breast during Pregnancy and Lactation}

The breast undergoes various changes during pregnancy and lactation in response to hormones such as estrogen, progesterone, and prolactin [1]. During pregnancy, the number and size of breast ducts and lobules increase. The fluid content of the breast also increases, accompanied by involution of the fibrofatty stroma $[1,5]$. After delivery, prolactin induces milk accumulation and corresponding lobular growth and distension, and oxytocin induces the milk ejection reflex and facilitates the maintenance of milk production $[1,4-6]$. These physiological changes result in increased breast volume with associated palpable nodularity and increased firmness, making the clinical detection of palpable lesions difficult [5]. Spontaneous unilateral or bilateral bloody nipple discharge can occur during pregnancy and early lactation (in up to $20 \%$ of pregnant women and $15 \%$ of lactating women)

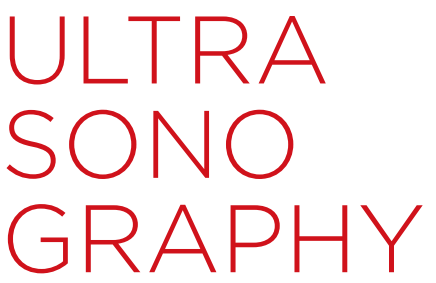

\section{PICTORIAL ESSAY}

https://doi.org/10.14366/usg. 19070 pISSN: 2288-5919 • elSSN: 2288-5943

Ultrasonography 2020;39:298-310

Received: November 6, 2019

Revised: January 3, 2020

Accepted: January 23, 2020

Correspondence to:

Seung Eun Lee, MD, Department of Radiology, Yeungnam University College of Medicine, 170 Hyeonchungro, Nam-gu, Daegu 42415, Korea

Tel. +82-53-620-4129

Fax. +82-53-653-5484

E-mail: air1986@naver.com

This is an Open Access article distributed under the terms of the Creative Commons Attribution NonCommercial License (http://creativecommons.org/ licenses/by-nc/4.0/) which permits unrestricted noncommercial use, distribution, and reproduction in any medium, provided the original work is properly cited.

Copyright (C) 2020 Korean Society of Ultrasound in Medicine (KSUM)

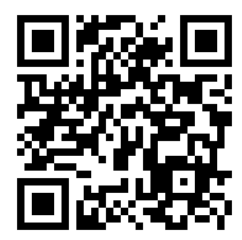

How to cite this article:

Lee SE, Bae YK. Breast lesions during pregnancy and lactation: a pictorial essay. Ultrasonography. 2020 Jul;39(3):298-310. 
due to proliferative changes in epithelial cells as well as increased breast vascularity $[1,4]$. This condition is usually self-limiting. However, persistent unilateral bloody nipple discharge can be a pathological condition that occurs secondary to infection, papilloma, or breast cancer. A diagnostic imaging evaluation is recommended if pathological nipple discharge is suspected $[1,4,7]$.

\section{Imaging Evaluations and Breast Biopsies during Pregnancy and Lactation}

The evaluation of breast imaging studies during pregnancy and lactation is challenging because of physiological and structural changes in the breast, as well as the need to balance maternal and fetal well-being [7].

\section{Mammography}

On mammography, physiological changes during pregnancy and lactation result in a diffuse increase in parenchymal density with breast enlargement (Fig. 1). These changes decrease the sensitivity of mammography by $70 \%-90 \%[1,4-6]$.

Mammography is generally safe during pregnancy, as it exposes the mother and fetus to negligible radiation. The fetal radiation dose from a four-view mammogram is less than $0.03 \mathrm{mGy}$ and does not have teratogenic effects $[8,9]$. In their Appropriateness Criteria, the American College of Radiology (ACR) established evidence-based guidelines for specific clinical conditions. According to these guidelines, there is limited evidence to support breast cancer screening in pregnant or lactating women. However, screening mammography is not contraindicated during pregnancy, particularly in women at intermediate to high risk for breast cancer. Mammography and digital breast tomosynthesis are appropriate modalities for breast cancer screening. In a diagnostic setting, mammography can be used as a supplement to ultrasonography for breast evaluations in pregnant women with palpable masses or pathological nipple discharge. A complete mammographic evaluation is recommended for locoregional staging in pregnant women with newly diagnosed breast cancer $[7,9]$.

\section{Ultrasonography}

On ultrasonography, during pregnancy, the breast shows diffuse hypoechogenicity with fibroglandular enlargement and increased vascularity (Fig. 2A). In lactating women, the breast shows diffuse hyperechogenicity with a prominent ductal system and increased vascularity (Fig. 2B, C) $[4,6,10,11]$. Ultrasonography is the best

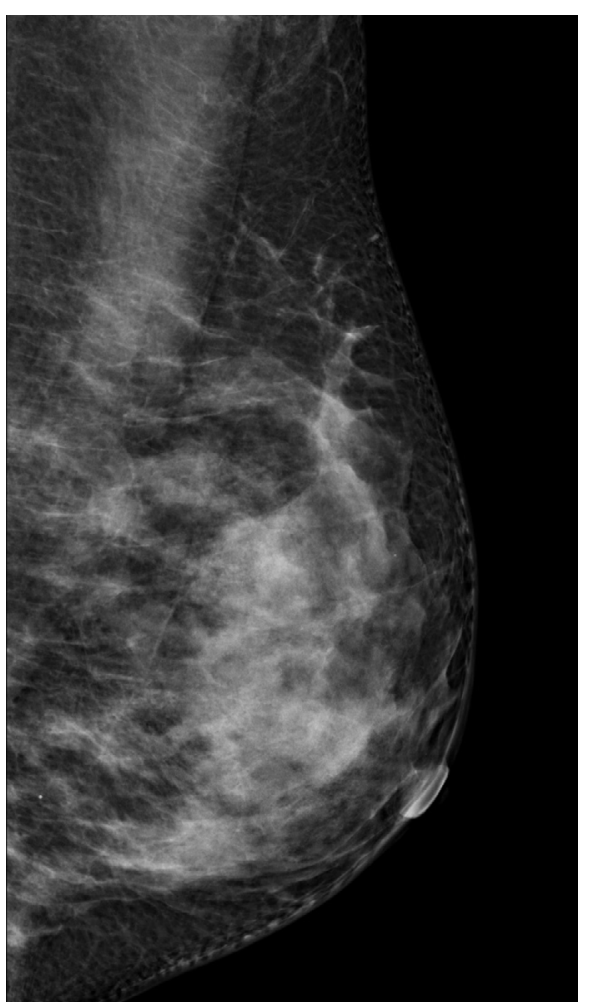

A

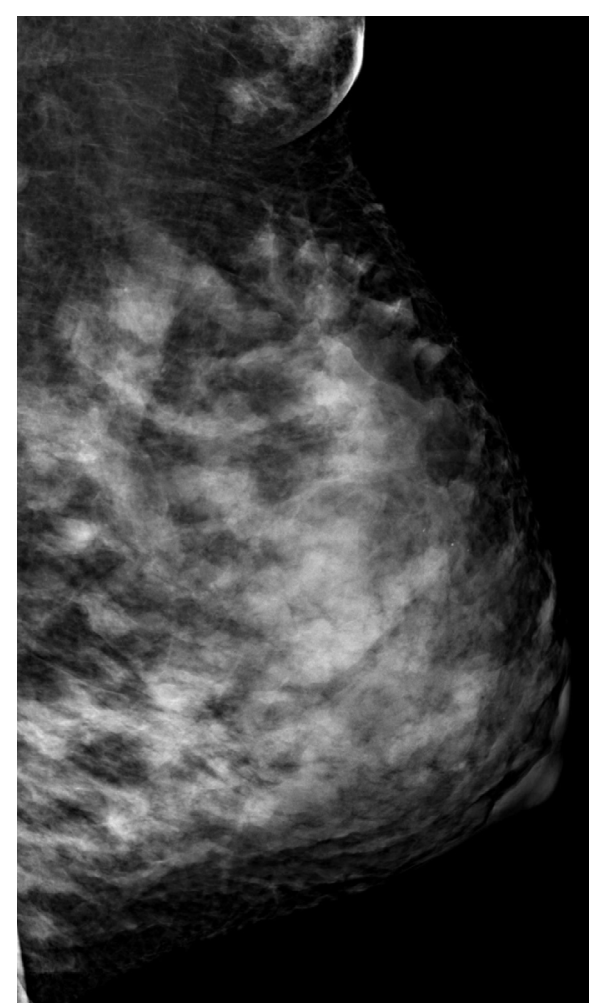

B
Fig. 1. Mammography of physiological changes in a 33-year-old lactating woman.

A. A baseline mammogram obtained before pregnancy shows a heterogeneously dense breast. B. Screening mammography performed during lactation shows breast enlargement and increased parenchymal density with an increased amount of glandular tissue and accessory breast tissue in the axilla. 
imaging modality to evaluate breast lesions during pregnancy and lactation, as it is sensitive and confers no radiation exposure $[6,11,12]$. According to the ACR Appropriateness Criteria, pregnant woman with palpable masses or pathological nipple discharge should be initially evaluated by ultrasonography in order to characterize the features of the lesion and plan proper management $[1,7,13]$.

\section{Magnetic Resonance Imaging}

The physiological increases in breast vascularity during pregnancy and lactation result in markedly increased background parenchymal enhancement on breast dynamic contrast-enhanced magnetic resonance imaging (MRI). These changes may limit the sensitivity of MRI [14].

MRI has a limited role during pregnancy and lactation due to concerns about gadolinium exposure and limited data regarding the safety of the technique for such use [8]. According to the ACR Appropriateness Criteria, the routine use of breast MRI is

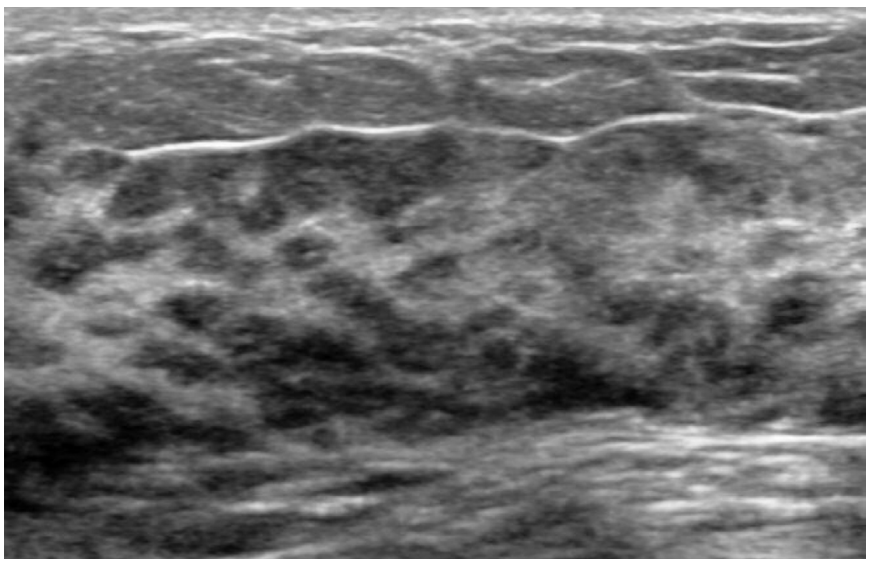

A

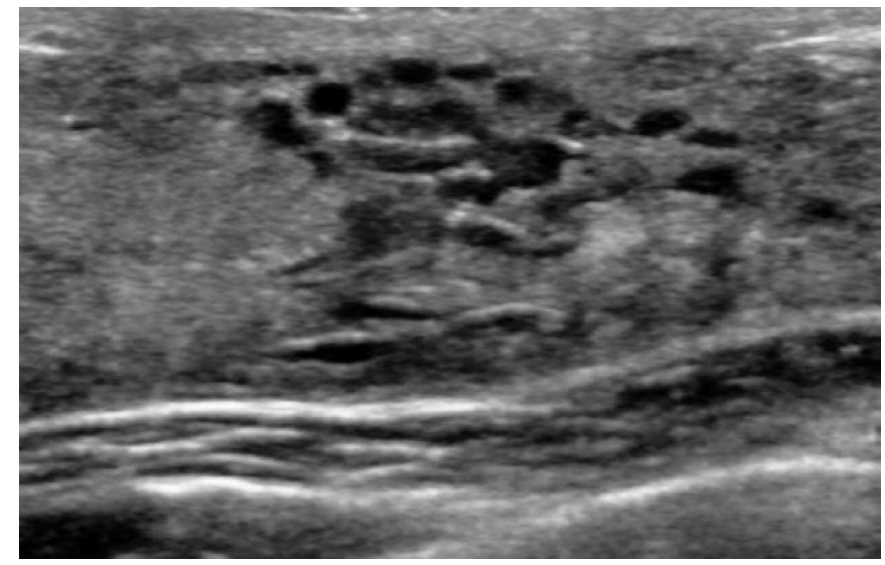

C not appropriate in either screening or diagnostic settings during these periods; rather, use of the technique is recommended only in situations where the risk-benefit ratio is clear [7].

\section{Core Needle Biopsy}

Core needle biopsy is considered safe during pregnancy and lactation, but caution should be exercised due to the relatively high risk of bleeding, infection, or milk fistula formation $[1,8]$. To minimize complications, patients are recommended to discontinue breastfeeding prior to biopsy. Additionally, procedures should be performed only under strict aseptic conditions. Moreover, it is helpful to monitor and facilitate hemostasis by using compression or ice packs after biopsy [1].

\section{Inflammatory and Infectious Diseases}

\section{Puerperal Mastitis}

Mastitis occurs relatively often during lactation, specifically in up

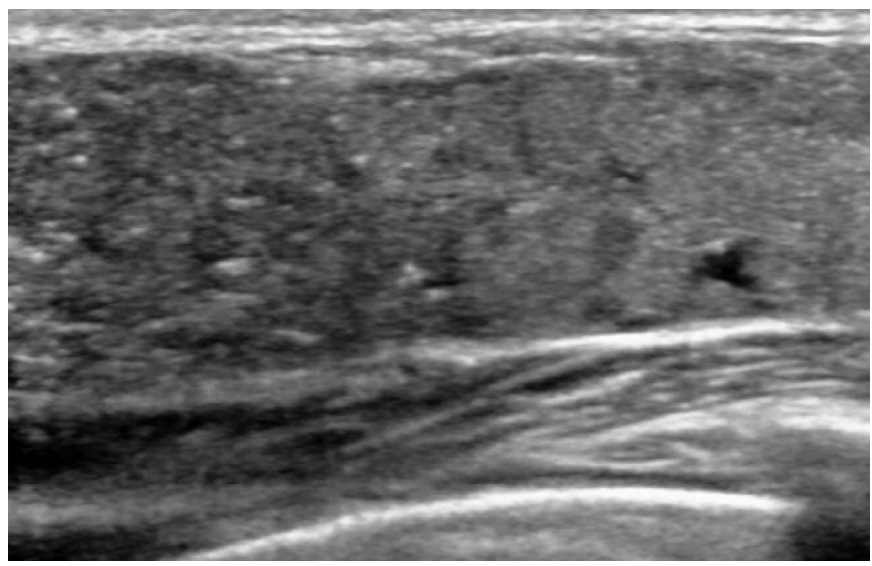

B

Fig. 2. Ultrasonography of physiological changes during pregnancy and lactation.

A. Ultrasonography of a 34-year-old pregnant woman (intrauterine pregnancy, 23 weeks) shows diffuse enlargement of the nonfatty glandular component with diffuse hypoechogenicity. B, C. Ultrasonography of a 30-year-old lactating woman show diffuse enlargement of the glandular component with multiple hyperechogenic dots and a prominent ductal system with ductal dilatation. 
to $20 \%-25 \%$ of breastfeeding women [15]. The most common pathogens are Staphylococcus aureus and Streptococcus, which are present in the infant's nasopharynx and infect the breast through nipple cracks during breastfeeding [4]. If an abscess is suspected, ultrasonography is the most useful modality for the diagnosis and treatment of mastitis $[1,4]$. On ultrasonography, mastitis appears as an ill-defined area of altered echotexture, usually with hypoechoic or heterogeneous echogenicity; increased vascularity is observed on color Doppler ultrasonography (Fig. 3). Ancillary findings include skin thickening and enlarged axillary lymph nodes [2]. The abscess is an irregular hypoechoic or anechoic mass, sometimes with internal echogenic debris and/or posterior acoustic enhancement (Fig. 4) [6]. Treatment of mastitis includes appropriate antibiotic therapy.
Ultrasound-guided fine-needle aspiration, vacuum-associated biopsy, or surgical drainage can be effective treatment options in the case of abscess formation $[15,16]$. However, if the patient does not respond to antibiotic therapy for puerperal mastitis, the possibility of cancer should be considered and further evaluation should be performed $[2,4]$.

\section{Granulomatous Mastitis}

Granulomatous mastitis is a rare inflammatory disease of unknown etiology that typically affects young women within 5 years of pregnancy $[1,6]$. Clinically, it manifests as a firm to hard mass and is accompanied by reactive lymphadenopathy in up to $15 \%$ of patients $[1,2]$. On ultrasonography, solitary or multiple clustered, circumscribed, or irregular heterogeneous hypoechoic masses are

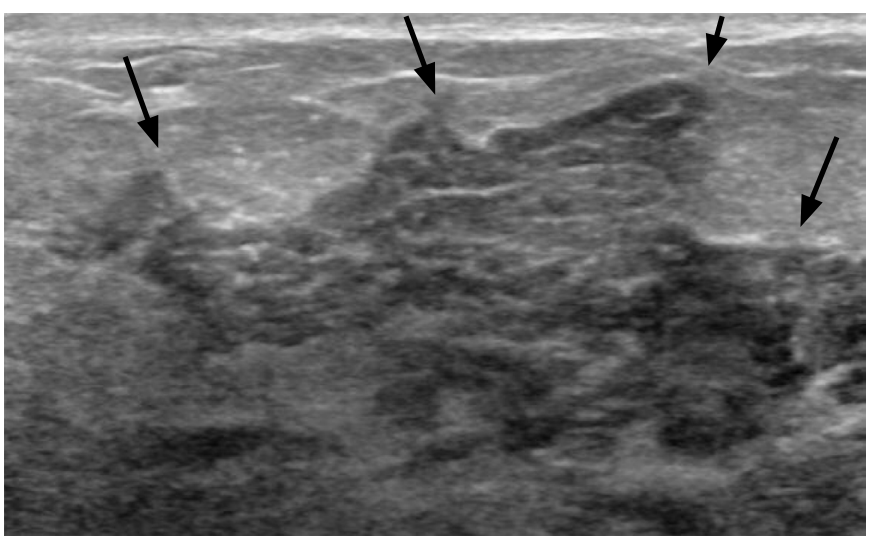

A

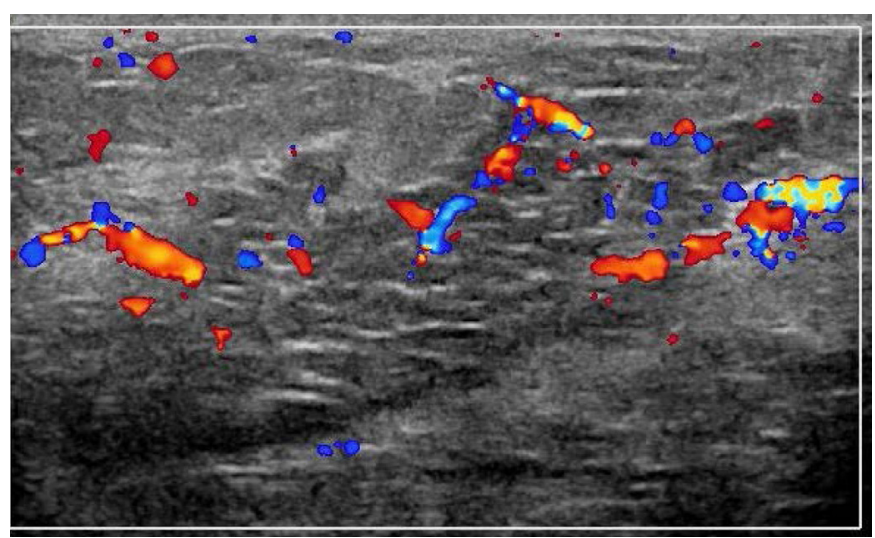

B

Fig. 3. Puerperal mastitis in a 28-year-old lactating woman with right breast pain and fever.

A. Ultrasonography shows engorgement of the right breast with irregular, partially indistinct, hypoechoic, and heterogeneous areas (arrows). B. Color Doppler ultrasonography shows increased vascularity.

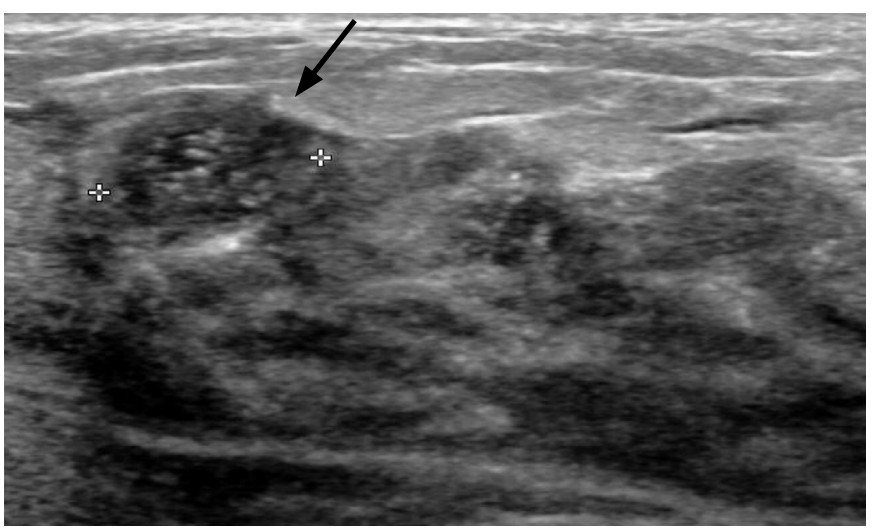

A

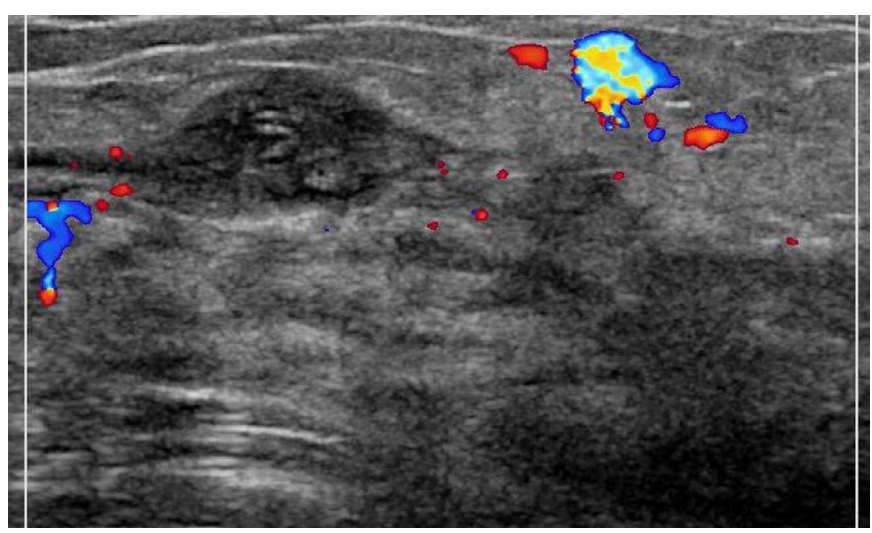

B

Fig. 4. Puerperal mastitis with abscess in a 32-year-old lactating woman who reported having a palpable mass for 4 months.

A. Ultrasonography shows poorly defined amorphous areas of hypoechoic and heterogeneous echogenicity, suggesting mastitis. The abscess is an oval-shaped, complex heterogeneous mass with hyperechoic debris (arrow). B. Color Doppler ultrasonography shows perilesional vascularity. Purulent exudate was obtained with fine-needle aspiration. 
observed, often with contiguous tubular configurations (Fig. 5). Diffuse abscess or fistula formation can also be seen [13]. These masses can occur in any part of the breast but tend to spare the subareolar regions $[1,2]$. Due to the variations in imaging results, they can sometimes resemble malignancy. It is important to correlate the imaging features with the histopathological findings and to engage in long-term follow-up [13].

\section{Benign Disorders during Pregnancy and Lactation}

\section{Galactocele}

Galactocele is a milk-filled retention cyst caused by the obstruction of the lactiferous duct. It is the most common benign breast mass in women who are or who recently stopped lactating $[6,10]$. Clinically, it manifests as one or multiple painless palpable masses [10]. On mammography, galactocele can exhibit various imaging features depending on the proportion of fat and protein in the individual's breast milk. When completely radiolucent with a relatively high fat content, it is known as pseudolipoma. A cystic mass with a fat-fluid

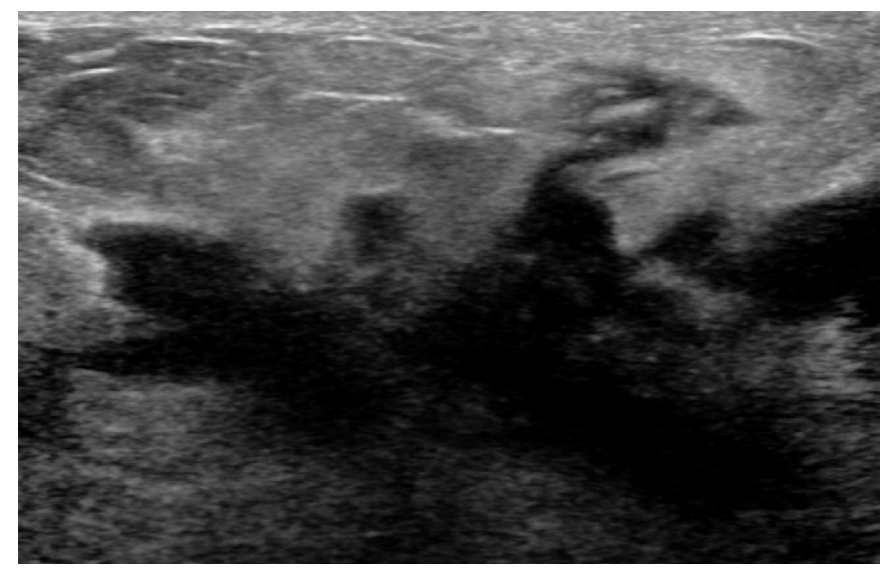

A

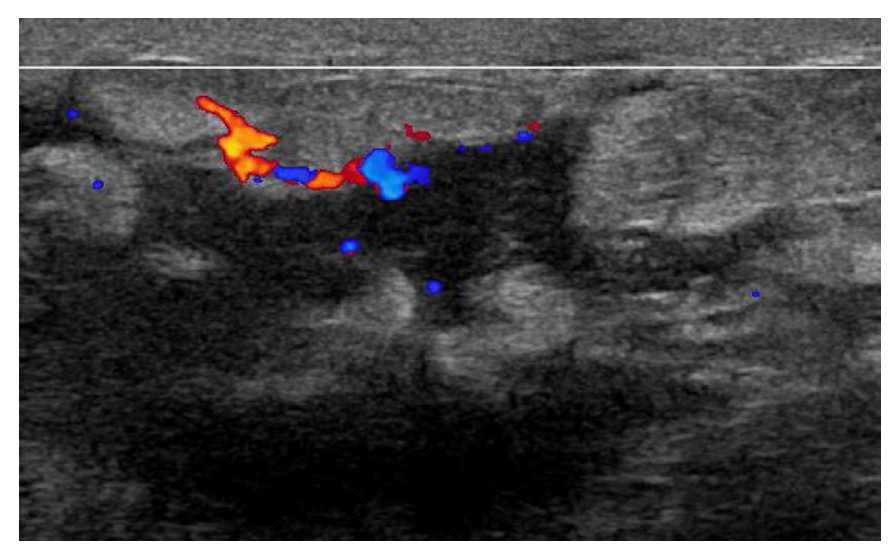

C level is a diagnostic indicator of a galactocele, with the fat content above and the water content remaining at the bottom. Alternatively, when the high viscosity of breast milk does not allow the separation of the fat and water contents, a galactocele can resemble a hamartoma; this condition is known as pseudohamartoma $[1,4,10]$. On ultrasonography, galactocele manifests as a simple or complicated cyst, typically an oval-shaped mass with a circumscribed margin and variable internal echogenicity (Fig. 6) $[1,10,17]$. A common complication of galactocele is infection. Galactocele infection is confirmed when mixed milky and purulent material is obtained by fine-needle aspiration. On ultrasonography, an infected galactocele appears relatively conspicuous and similar to an abscess (Fig. 7) [1]. Uncomplicated galactoceles with typical imaging features are self-limiting; in cases of galactoceles with associated symptoms or atypical imaging features, ultrasound-guided fineneedle aspiration should be performed [10].

\section{Lactating Adenoma}

A lactating adenoma is a benign tumor that develops in response to physiological hormonal changes during pregnancy and lactation

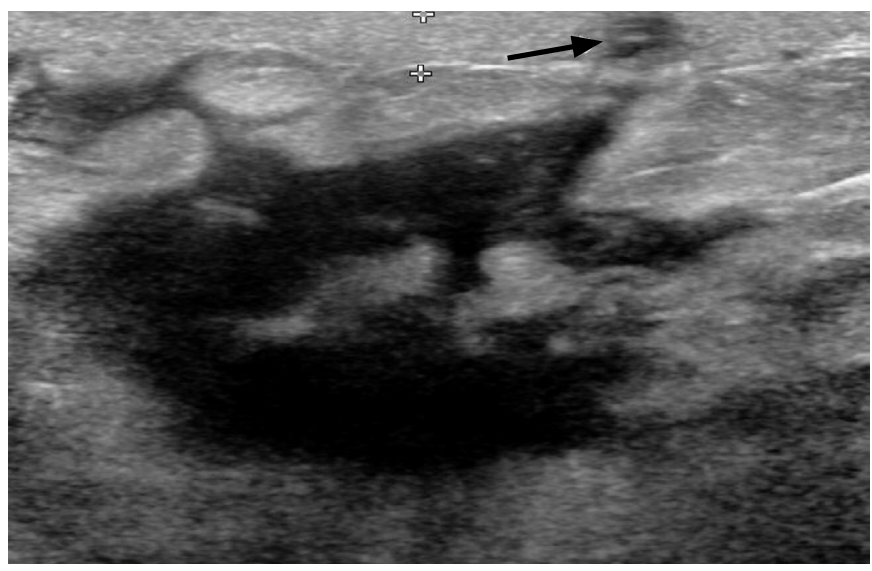

B

Fig. 5. Granulomatous mastitis in a 37-year-old pregnant woman (intrauterine pregnancy, 17 weeks) who presented with breast pain.

A, B. Ultrasonography show irregular hypoechoic lesions with tubular extensions to the subcutaneous tissues and skin arrow in B. Associated changes, such as overlying skin thickening and increased echogenicity of the surrounding fat tissue, are also visible. C. Color Doppler ultrasonography shows perilesional vascularity. Ultrasoundguided core needle biopsy reveals granulomatous lobular mastitis. 


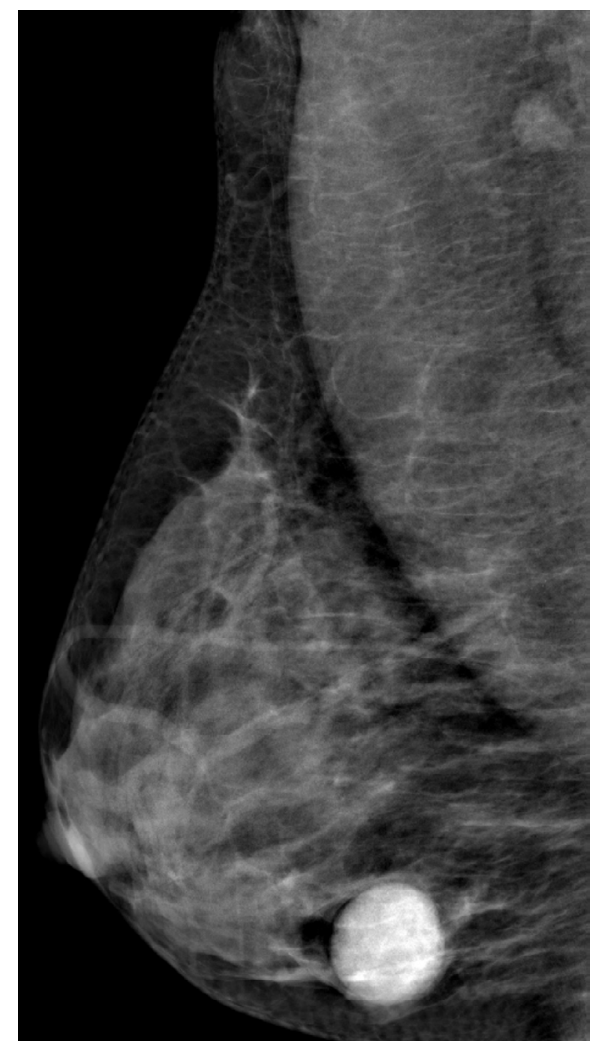

A

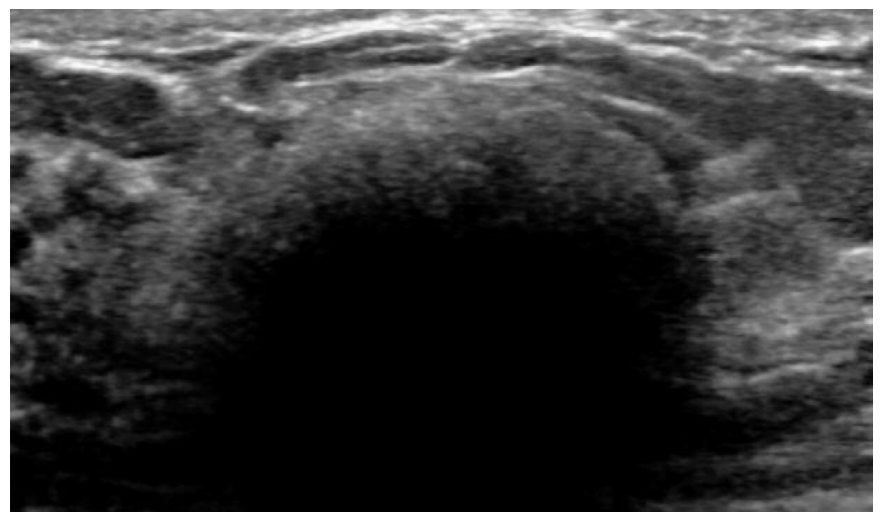

B

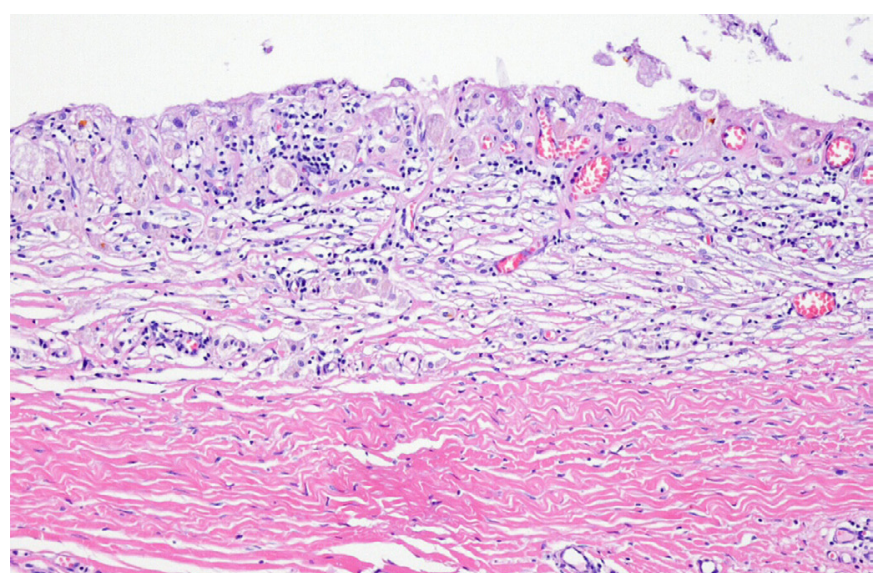

C

Fig. 6. Galactocele in a 40-year-old woman with a palpable mass.

The palpable mass was detected during lactation and examined 6 months after cessation of breastfeeding. A. A mediolateral oblique mammogram shows an oval-shaped, circumscribed hyperdense mass. B. Ultrasonography shows an oval-shaped mass with echogenic contents and posterior acoustic shadowing. Ultrasound-guided core needle biopsy and subsequent excision were performed. C. The lining epithelium of cystically dilated duct is desquamated. Chronic inflammatory changes with lymphohistiocytic infiltration and fibrosis in the wall can be observed $(H \& E, \times 100)$. Considering the clinical history and input from a pathologist, the lesion was hypothesized to have originated as a galactocele relatively long ago. It was accompanied by chronic inflammation secondary to leakage.

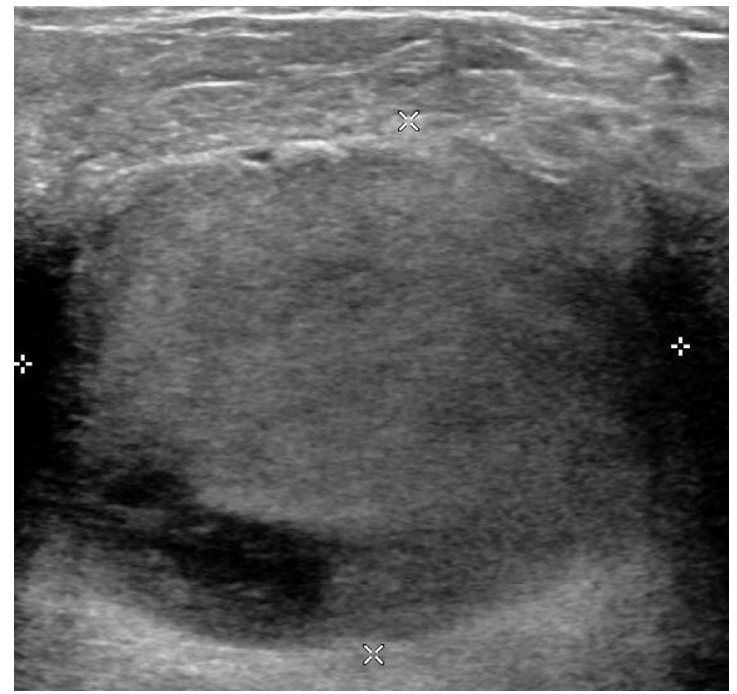

Fig. 7. Infected galactocele in a 31-year-old lactating woman with a palpable mass. Ultrasonography shows an oval-shaped, circumscribed, complex cystic mass with posterior acoustic enhancement. Fine-needle aspiration reveals purulent and milky material, confirming the presence of a clinically infected galactocele. 
[10]. Histopathologically, lactating adenomas display lobular expansion with numerous enlarged and dilated acini separated by connective tissue. Lactating adenoma has been suggested to be a

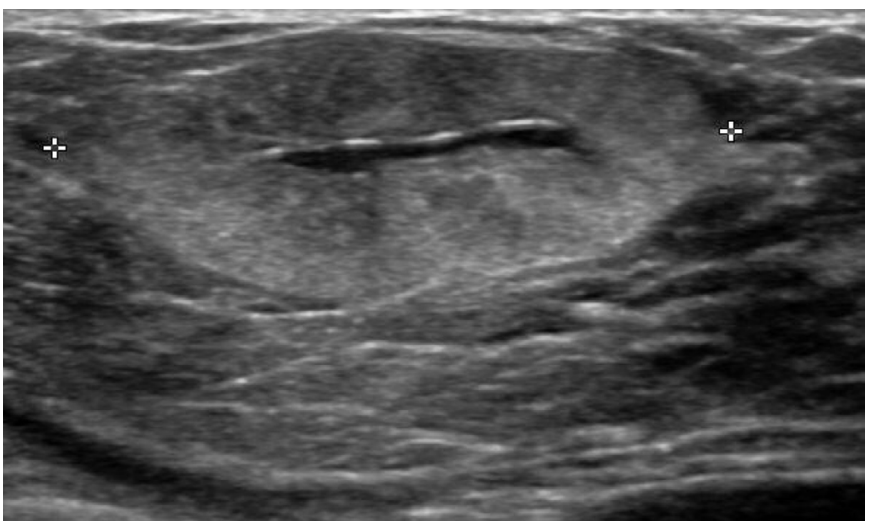

A

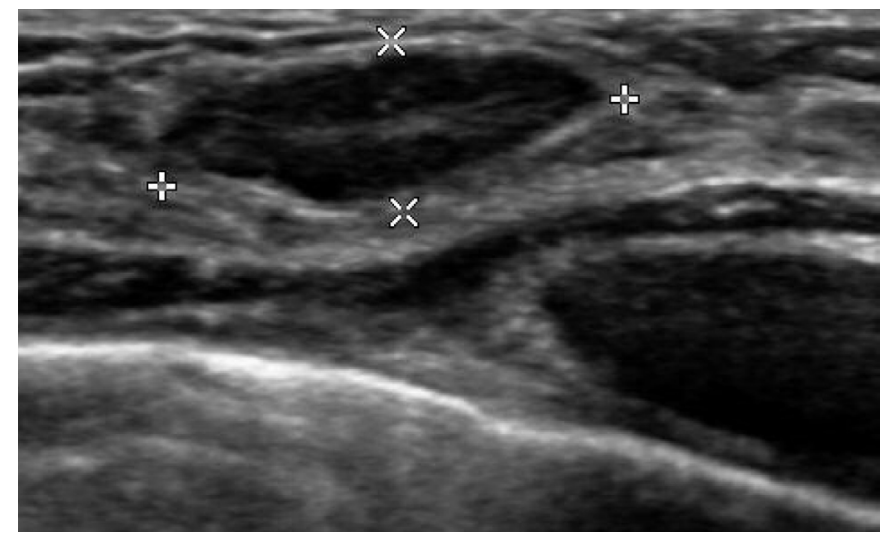

C

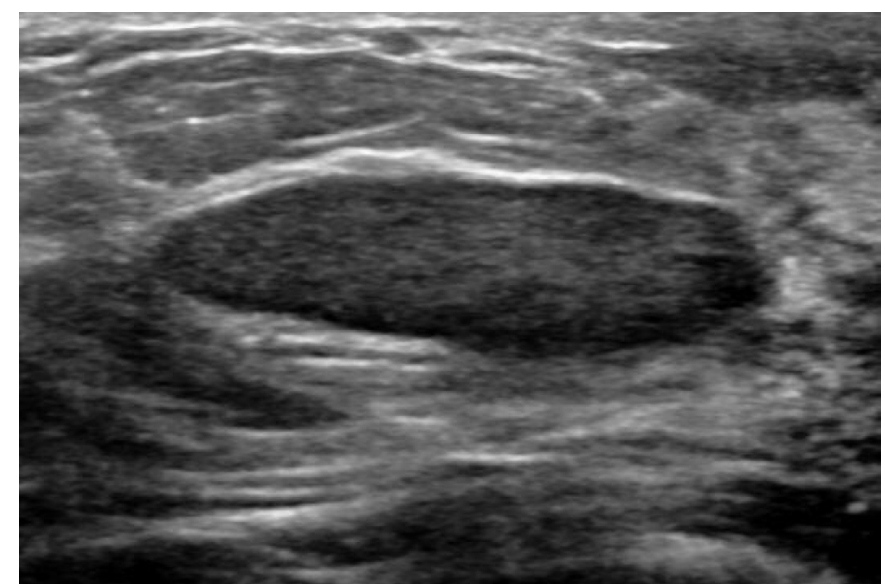

A variant of fibroadenoma, tubular adenoma, or lobular hyperplasia $[6,10]$, and it can spontaneously regress after pregnancy and lactation [10]. On ultrasonography, lactating adenomas usually

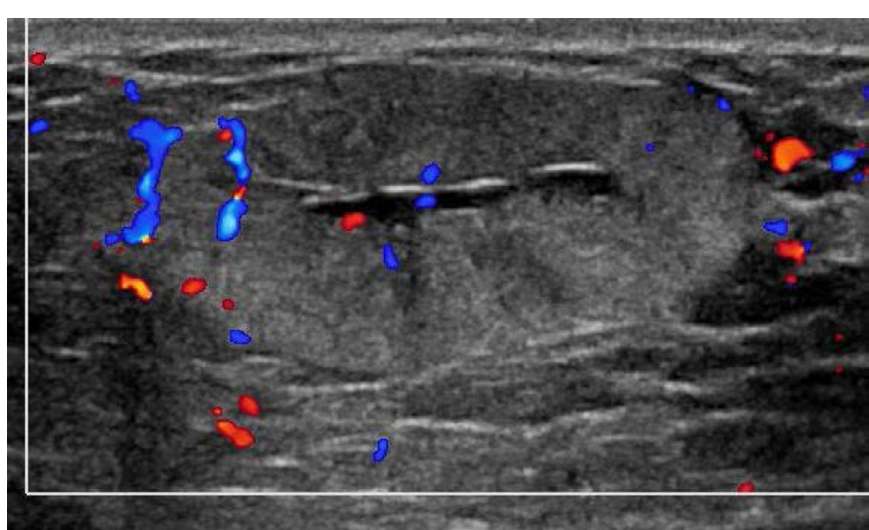

B

Fig. 8. Lactating adenoma in a 29-year-old lactating woman with a palpable mass.

A. Ultrasonography shows an oval, circumscribed hyperechoic mass with a central hypoechoic area and posterior acoustic enhancement. B. Color Doppler ultrasonography shows internal vascularity. Ultrasound-guided core needle biopsy revealed a lactating adenoma. C. On a follow-up ultrasonography 8 months after cessation of lactation, the lactating adenoma had decreased in both size and internal echogenicity.

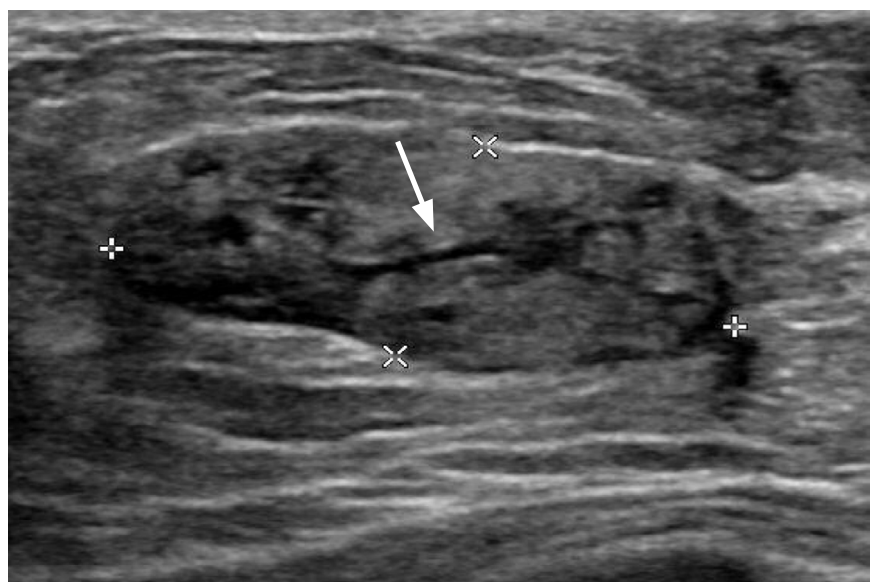

B

Fig. 9. Fibroadenoma with lactational changes in a 36-year-old lactating woman.

A. Ultrasonography obtained before pregnancy shows an oval, circumscribed, hypoechoic mass, which a biopsy showed to be fibroadenoma. B. In the lactational period, the pre-existing fibroadenoma changed in response to hormonal stimulation. The fibroadenoma shows mixed heterogeneous echogenicity with a prominent ductal pattern (arrow). 


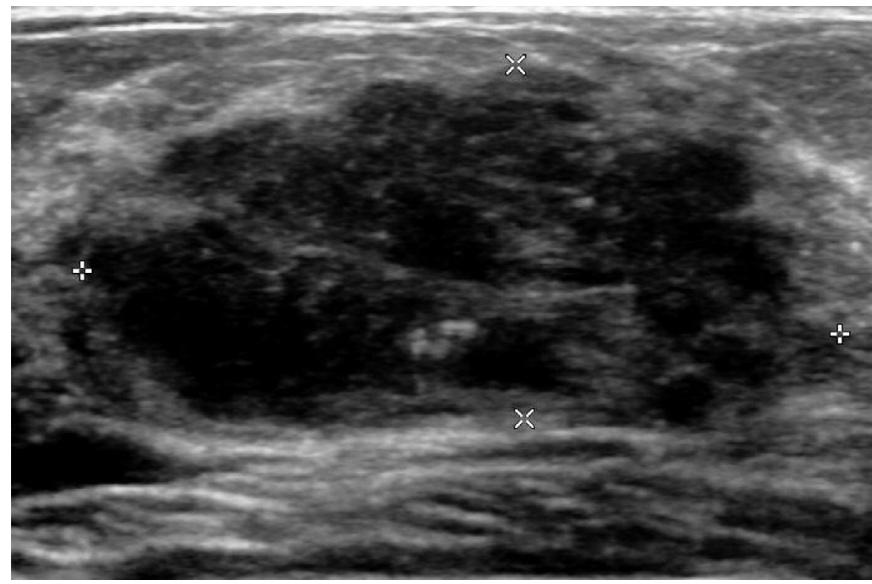

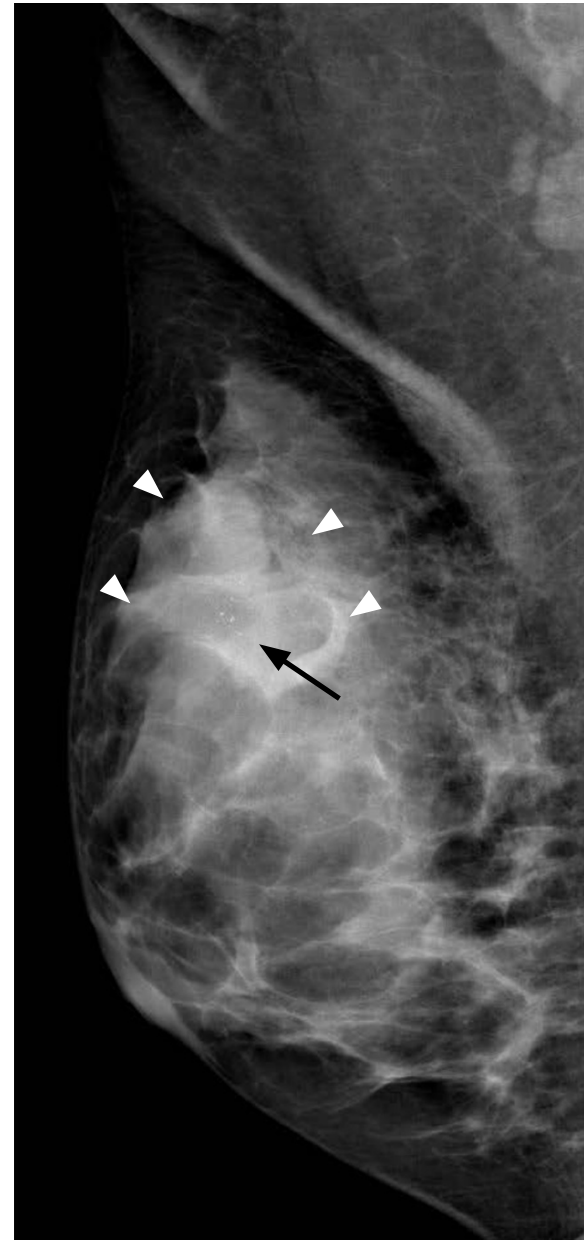

A

B

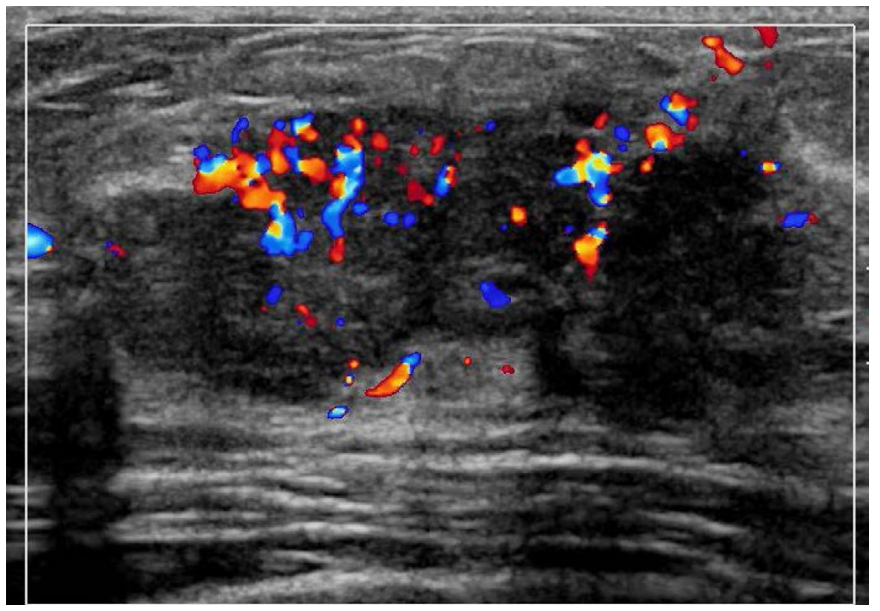

C

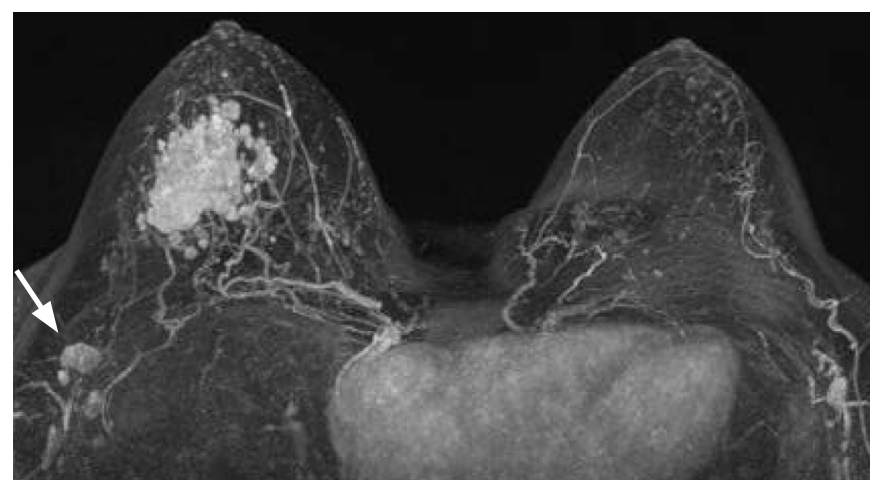

D

Fig. 10. Pregnancy-associated breast cancer in a 37-year-old woman (8 months after delivery) presenting with a palpable mass in the right breast.

A. A mediolateral oblique mammogram shows focal asymmetry (arrowheads) with grouped amorphous microcalcifications (arrow) in the right upper outer quadrant. The lesion is partially obscured by dense parenchymal tissue. B. Gray-scale ultrasonography shows an irregular-shaped, hypoechoic mass with a microlobulated margin and calcifications. C. Color Doppler ultrasonography shows intratumoral hypervascularity, and core needle biopsy revealed invasive ductal carcinoma. D. A post-contrast T1-weighted maximum intensity projection image (TR/TE 5.3/1.9) shows an irregular-shaped conglomerated mass, measuring $4 \mathrm{~cm}$ at the point of longest diameter, in the right upper outer quadrant of the breast; additionally, axillary metastatic lymphadenopathies are visible (arrow). The patient underwent right breastconserving surgery after neoadjuvant chemotherapy. 
manifest as oval, circumscribed, homogeneous, hypoechoic or isoechoic masses and/or posterior acoustic enhancements (Fig. 8A, B). However, lesions with atypical imaging features such as irregular margins, heterogeneity, or posterior acoustic shadowing may mimic malignancy $[1,10]$. Lactating adenomas can regress naturally upon cessation of pregnancy or lactation (Fig. 8C) [4].

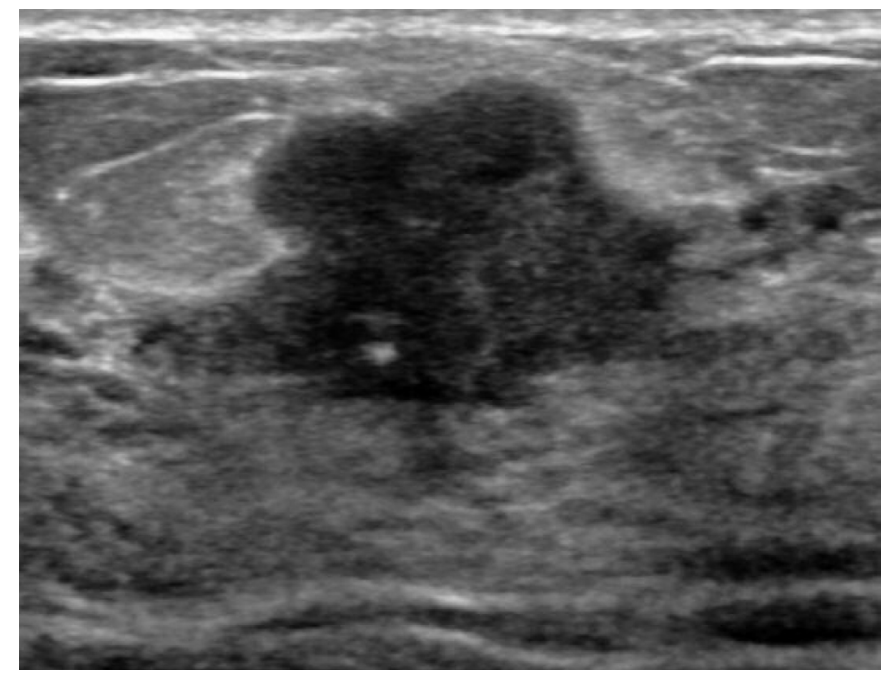

\section{Fibroadenoma}

Fibroadenomas are the most common benign breast tumors in young women. The image features of fibroadenomas are usually similar in pregnant and non-pregnant woman [2]. However, morphological and physiological changes can occur during pregnancy and lactation. As hormone-sensitive tumors,

Fig. 11. Pregnancy-associated breast cancer with local recurrence and distant metastases in a 30-year-old pregnant woman (intrauterine pregnancy, 37 weeks).

A. Ultrasonography shows an irregular-shaped, hypoechoic mass with an angular margin. The patient underwent right breastconserving surgery after delivery, and the histologic findings revealed invasive ductal carcinoma. B-E. Local recurrence and distant metastases occured 30 months after breast-conserving surgery for initial pregnancy-associated breast cancer. C. Ultrasonography shows a newly developed, irregular hypoechoic mass at the surgical site, demonstrated by core needle biopsy to be recurrent invasive ductal carcinoma. Positron emission tomography-computed tomography and chest computed tomography indicate the presence of lung and bone metastases $(B, D)$, and brain magnetic resonance imaging indicates the presence of brain metastasis (E).

A

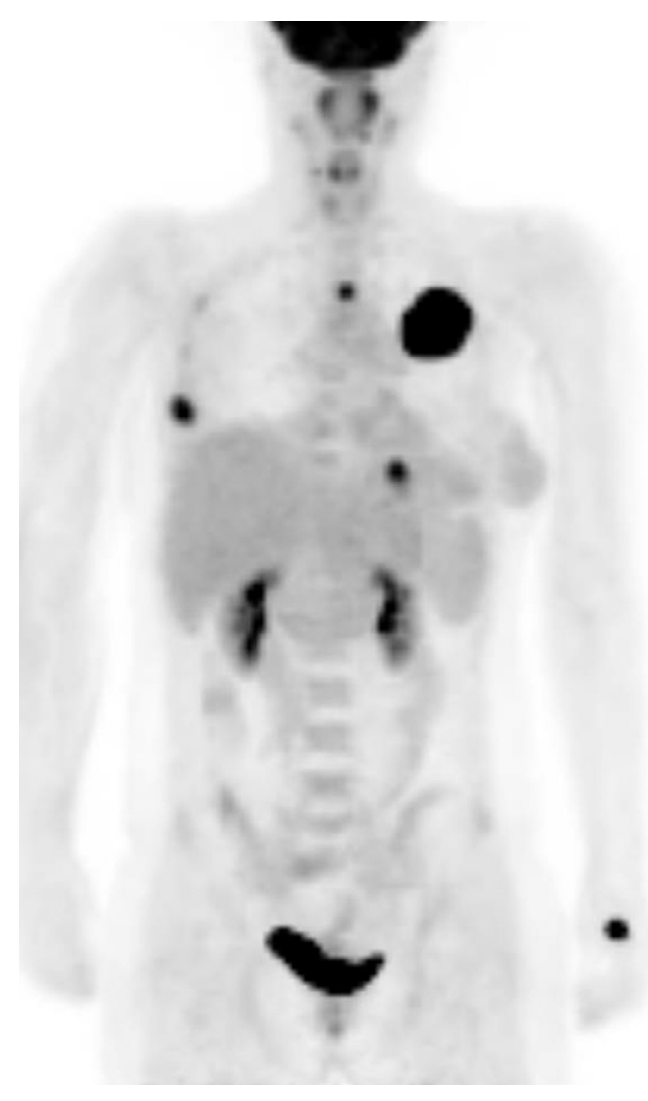

B

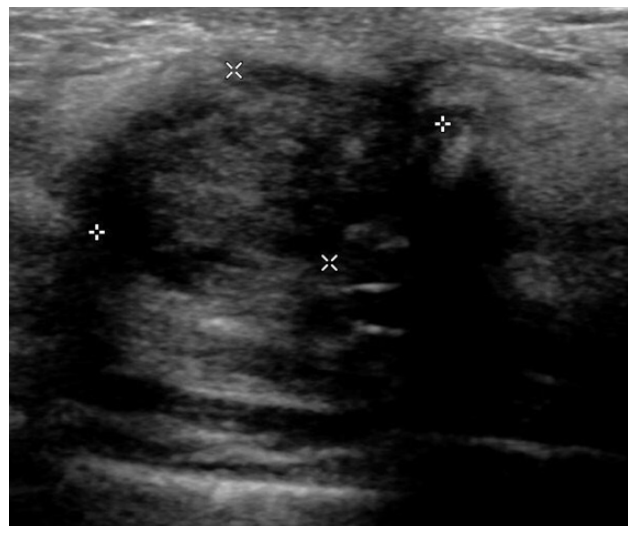

C

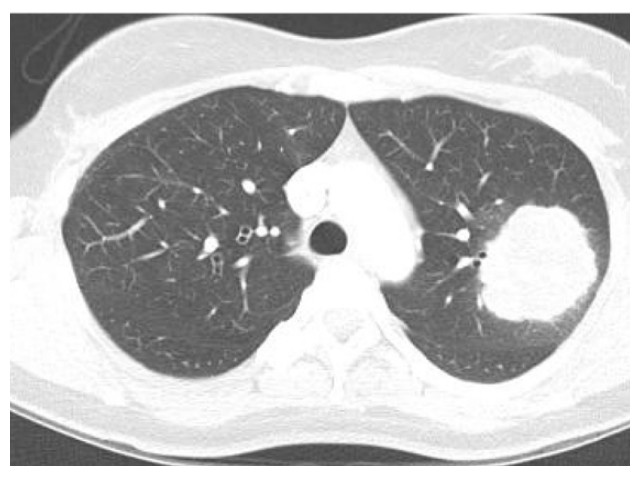

D

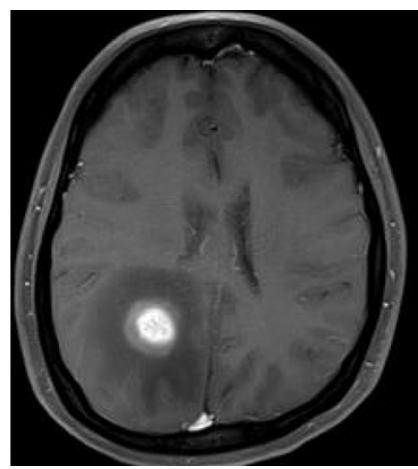

$\mathrm{E}$ 
fibroadenomas often become enlarged with or without increased vascularity during pregnancy and lactation, and they can regress after the cessation of breastfeeding $[1,13]$. Spontaneous infarction of fibroadenoma is very rare, but can sometimes occur during pregnancy or lactation. Infarction can cause a fibroadenoma to exhibit more complex features, such as lobulated margins, heterogeneous echotexture, and posterior acoustic shadowing $[1,4]$. Secretory hyperplasia can sometimes occur in fibroadenomas during pregnancy and lactation. Fibroadenoma with secretory hyperplasia or lactational changes can exhibit heterogeneous echotexture and/ or dilated ducts and cysts, resembling a complex fibroadenoma (Fig. 9) [1]. In cases with atypical radiological features, percutaneous core

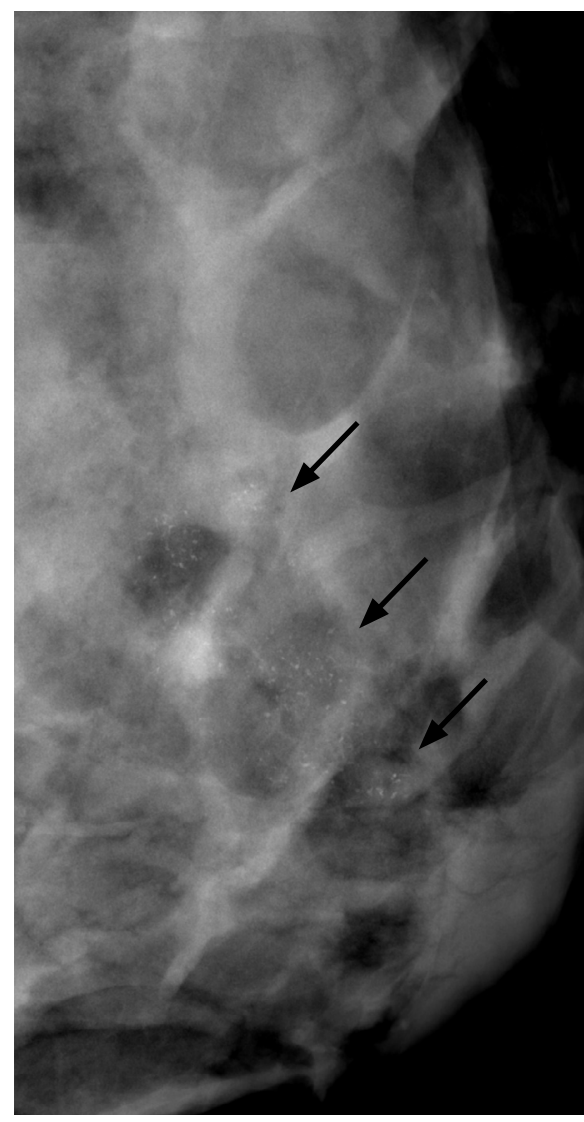

A

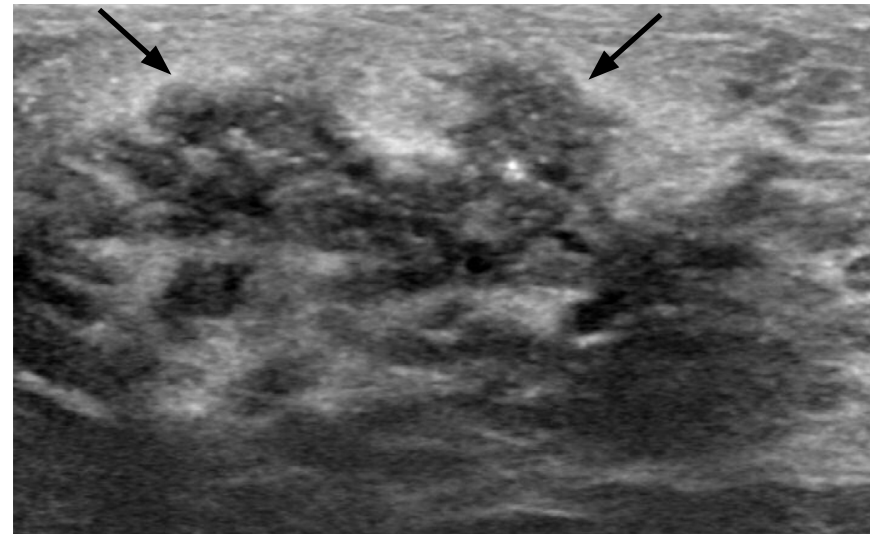

B

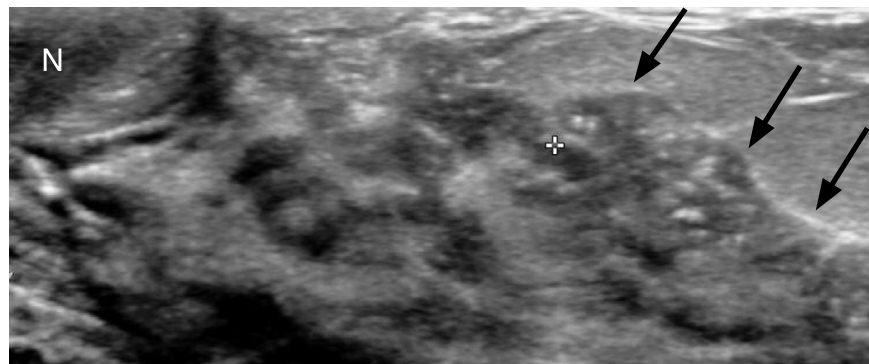

C

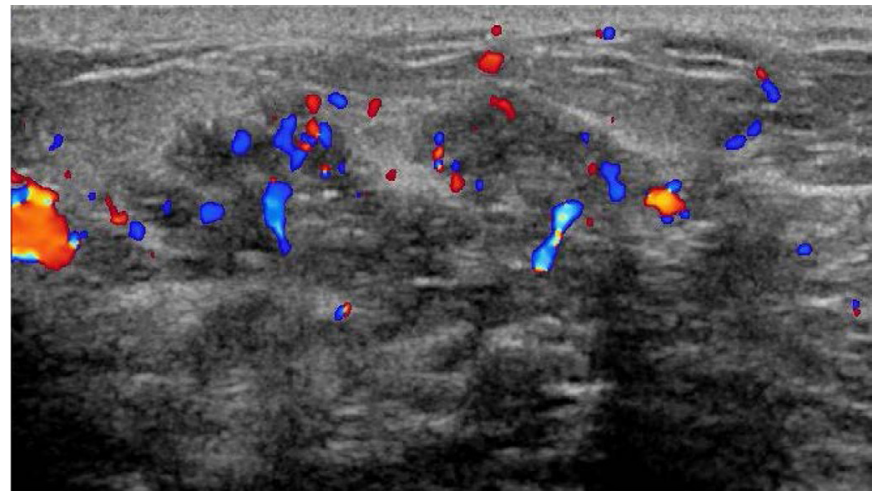

D

Fig. 12. Pregnancy-associated breast cancer in a 42-year-old pregnant woman (intrauterine pregnancy 35 weeks) presenting with bloody nipple discharge.

A. Mediolateral oblique mammogram shows segmental distributed, fine pleomorphic microcalcifications (arrows). B, C. Ultrasonography shows an irregular ductal change with intraductal calcifications (arrows). D. Color Doppler study shows increased vascularity within the lesion. The patient underwent left breast conserving surgery and histologic findings revealed invasive carcinoma with ductal carcinoma in situ. 


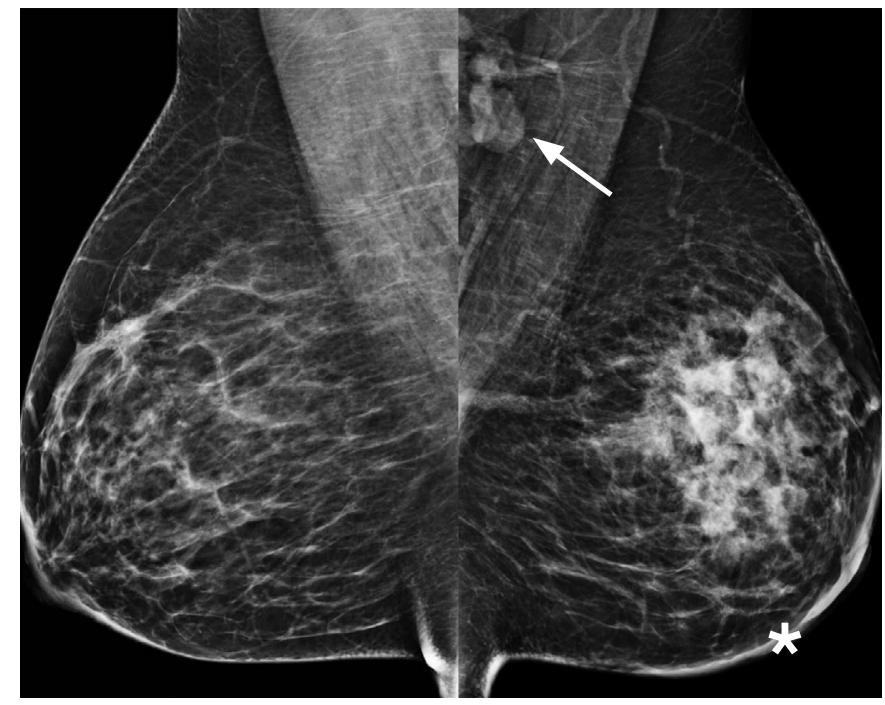

A

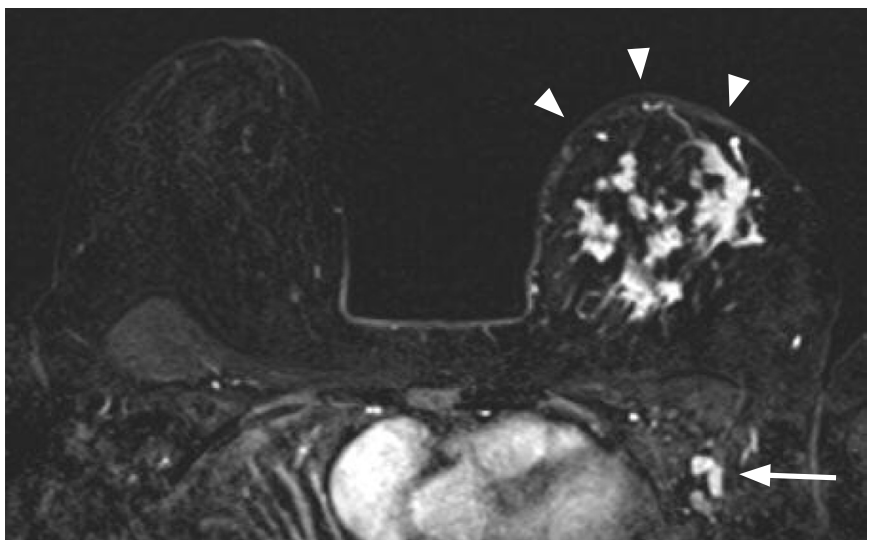

C

needle biopsy should be performed to confirm the diagnosis.

\section{Malignant Disorders during Pregnancy and Lactation}

\section{Pregnancy-Associated Breast Cancer}

Breast cancer that occurs during pregnancy or within 1 year of delivery is defined as PABC. PABC occurs in one of every 3,00010,000 pregnancies and accounts for $1 \%-3 \%$ of all breast malignancies $[4,5]$. PABC usually manifests as a painless, palpable mass. Because of the difficulty in carrying out clinical examinations during pregnancy and shortly afterward, diagnosis may be delayed $[2,4]$. PABCs are biologically aggressive and have been found to be larger and more advanced than non-PABCs in same-age patients $[2,5]$. More than $50 \%$ of patients present with axillary lymph node metastasis; additionally, a high prevalence of hormone-receptor-

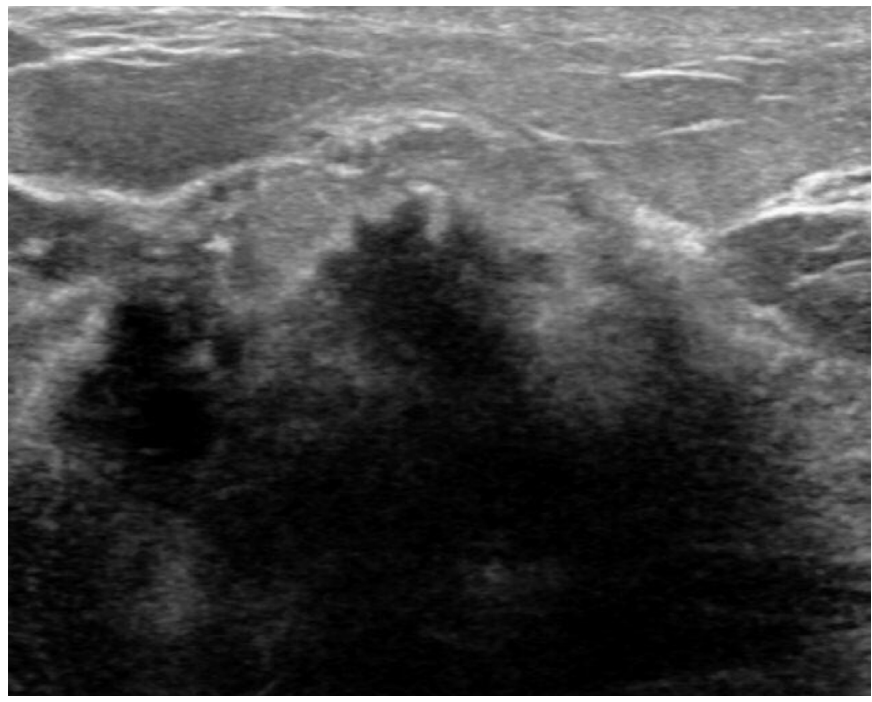

B

Fig. 13. Pregnancy-associated breast cancer in a 30 -year-old woman (10 months postpartum) with a palpable mass in her left breast for the prior 2 months.

A. Bilateral mediolateral oblique mammography show global asymmetry with multiple irregular-shaped masses and diffuse skin thickening (asterisk) in the left breast. Enlarged lymph nodes are also seen in the left axilla (white arrow). B. Ultrasonography shows multiple irregular-shaped masses with posterior acoustic shadowing. C. Post-contrast T1-weighted positron emission tomographymagnetic resonance image shows multiple irregular enhancing masses with diffuse skin thickening (arrowheads) in the left breast and enlarged lymph nodes (arrow) in the left axilla. These findings are consistent with inflammatory breast cancer. Core needle biopsy revealed invasive ductal carcinoma.

negative and HER2/neu-positive tumors has been reported in PABC, as has a high rate of inflammatory breast cancer $[1,12,18]$ (Figs. 10, 11). A combination of delayed diagnosis and an aggressive growth pattern due to the biological effects of pregnancy results in poor prognosis; recurrence is common and appears within 2-3 years of diagnosis $[1,2,13]$. The imaging features of $P A B C$ are similar to those of non-PABC. The sensitivity of mammography is relatively low in pregnant or lactating patients due to increased glandular density; however, mammography may still be useful to visualize malignant microcalcifications, multifocality, multicentricity, or bilaterality [6] (Fig. 12). Ultrasonography is the most appropriate modality to assess PABC $[1,12]$. On ultrasonography, PABC manifests as a circumscribed to spiculated, hypoechoic to complex mass with or without posterior acoustic shadowing. However, aggressive tumors can show posterior acoustic enhancement that reflects necrotic or cystic degeneration. Associated features include thickening of the Cooper's ligaments, 
edema, skin thickening, and axillary lymphadenopathy [2]. On $M R I$, features of PABCs include circumscribed to irregular margins, heterogeneous or rim enhancement, segmental distributed nonmass enhancement, and diffuse unilateral enhancement [19]. Despite the increased background parenchymal enhancement and T2 signal heterogeneity during pregnancy and shortly afterward, MRI may be a reliable modality of evaluating the extent and severity of PABC (Fig. 13) $[5,19,20]$.

\section{Conclusion}

The imaging findings associated with breast lesions that arise during pregnancy and lactation do not differ greatly from those associated with similar lesions in non-pregnant women. However, it is difficult to utilize breast imaging during pregnancy and lactation due to hormone-induced physiological changes. To facilitate the accurate diagnosis of breast lesions during this period, it is important to understand the imaging findings associated with common breast lesions and physiological changes.

ORCID: Seung Eun Lee: https://orcid.org/0000-0001-6693-4752; Young Kyung Bae: https://orcid.org/0000-0002-6689-9413

\section{Author Contributions}

Conceptualization: Lee SE. Data acquisition: Lee SE, Bae YK. Data analysis or interpretation: Lee SE, Bae YK. Drafting of the manuscript: Lee SE. Critical revision of the manuscript: Lee SE. Approval of the final version of the manuscript: all authors.

\section{Conflict of Interest}

No potential conflict of interest relevant to this article was reported.

\section{Acknowledgments}

This study was supported by a research grant from Yeungnam University.

\section{References}

1. Sabate JM, Clotet M, Torrubia S, Gomez A, Guerrero R, de las Heras $\mathrm{P}$, et al. Radiologic evaluation of breast disorders related to pregnancy and lactation. Radiographics 2007;27 Suppl 1:S101-S124.

2. Joshi S, Dialani V, Marotti J, Mehta TS, Slanetz PJ. Breast disease in the pregnant and lactating patient: radiological-pathological correlation. Insights Imaging 2013;4:527-538.

3. Son EJ, Oh KK, Kim EK. Pregnancy-associated breast disease: radiologic features and diagnostic dilemmas. Yonsei Med J
2006; 47:34-42.

4. Yu JH, Kim MJ, Cho H, Liu HJ, Han SJ, Ahn TG. Breast diseases during pregnancy and lactation. Obstet Gynecol Sci 2013;56:143-159.

5. Vashi R, Hooley R, Butler R, Geisel J, Philpotts L. Breast imaging of the pregnant and lactating patient: imaging modalities and pregnancy-associated breast cancer. AJR Am J Roentgenol 2013;200:321-328.

6. Canoy JM, Mitchell GS, Unold D, Miller V. A radiologic review of common breast disorders in pregnancy and the perinatal period. Semin Ultrasound CT MR 2012;33:78-85.

7. Expert Panel on Breast Imaging, diFlorio-Alexander RM, Slanetz PJ, Moy L, Baron P, Didwania AD, et al. ACR Appropriateness Criteria ${ }^{\circledR}$ Breast Imaging of Pregnant and Lactating Women. J Am Coll Radiol 2018;15:S263-S275.

8. Saunders C, Taylor D, Ives A. The role of breast imaging during pregnancy and lactation in the diagnosis of breast malignancy. Radiographer 2012;59:119-123.

9. Tremblay E, Therasse E, Thomassin-Naggara I, Trop I. Quality initiatives: guidelines for use of medical imaging during pregnancy and lactation. Radiographics 2012;32:897-911.

10. Teberian I, Bhimani C, Sciotto M, Wilkes A, Germaine P. Breast masses in pregnancy and lactation. J Am Osteopath Coll Radiol 2019;8:5-16

11. Tirada N, Dreizin D, Khati NJ, Akin EA, Zeman RK. Imaging pregnant and lactating patients. Radiographics 2015;35:1751-1765.

12. Ahn BY, Kim HH, Moon WK, Pisano ED, Kim HS, Cha ES, et al. Pregnancy- and lactation-associated breast cancer: mammographic and sonographic findings. J Ultrasound Med 2003;22:491-497.

13. de Holanda AA, Goncalves AK, de Medeiros RD, de Oliveira AM, Maranhao TM. Ultrasound findings of the physiological changes and most common breast diseases during pregnancy and lactation. Radiol Bras 2016;49:389-396.

14. Espinosa LA, Daniel BL, Vidarsson L, Zakhour M, Ikeda DM Herfkens RJ. The lactating breast: contrast-enhanced MR imaging of normal tissue and cancer. Radiology 2005;237:429-436.

15. Cacala SR. Breast conditions during pregnancy and lactation: an understanding of unique breast conditions associated with pregnancy and lactation is essential for evaluation and management of breast problems in pregnant or lactating women. Contin Med Educ 2010;28:508-512.

16. Kang YD, Kim YM. Comparison of needle aspiration and vacuumassisted biopsy in the ultrasound-guided drainage of lactational breast abscesses. Ultrasonography 2016;35:148-152.

17. Lee YH, Park YH, Kwon TH. Ultrasonographic findings of breast diseases during pregnancy and lactating period. J Korean Radiol Soc 1995;33:443-447.

18. Liberman L, Giess CS, Dershaw DD, Deutch BM, Petrek JA. Imaging of pregnancy-associated breast cancer. Radiology 1994;191:245-248.

19. Ayyappan AP, Kulkarni S, Crystal P. Pregnancy-associated breast 
cancer: spectrum of imaging appearances. Br J Radiol 2010;83:529534.

20. Kim SJ, Lim HS, Baek JM, Seon HJ, Heo SH, Kim JW, et al. Breast
MRI in pregnancy-associated breast cancer. J Korean Soc Radiol 2017;76:191-197. 